\title{
NKp44 and natural cytotoxicity receptors as damage-associated molecular pattern recognition receptors
}

\section{Nathan C. Horton and Porunelloor A. Mathew*}

Department of Cell Biology and Immunology, Institute for Cancer Research, University of North Texas Health Science Center, Fort Worth, TX, USA

\section{Edited by:}

Rafael Solana, University of Cordoba, Spain

Reviewed by:

Hugh Thomson Reyburn, Spanish National Research Council, Spain

Chiara Romagnani, Deutsches

Rheuma-Forschungszentrum,

Germany

Alfonso Martin-Fontecha,

Boehringer-Ingelheim, Germany

*Correspondence:

Porunelloor A. Mathew, Department of Cell Biology and Immunology,

University of North Texas Health

Science Center at Fort Worth, 3500

Camp Bowie Blvd., Fort Worth, TX

76107-2699, USA

e-mail: porunelloor.mathew@

unthsc.edu
Natural killer (NK) cells are a key constituent of the innate immune system, protecting against bacteria, virally infected cells, and cancer. Recognition and protective function against such cells are dictated by activating and inhibitory receptors on the surface of the NK cell, which bind to specific ligands on the surface of target cells. Among the activating receptors is a small class of specialized receptors termed the natural cytotoxicity receptors (NCRs) comprised of NKp30, NKp46, and NKp44. The NCRs are key receptors in the recognition and termination of virally infected and tumor cells. Since their discovery over 10 years ago, ligands corresponding to the NCRs have largely remained elusive. Recent identification of the cellular ligands for NKp44 and NKp30 as exosomal proliferating cell nuclear antigen (PCNA) and HLA-B-associated transcript 3 (BAT3), respectively, implicate that NCRs may function as receptors for damage-associated molecular pattern (DAMP) molecules. In this review, we focus on NKp44, which surprisingly recognizes two distinct ligands resulting in either activation or inhibition of NK cell effector responses in response to tumor cells. The inhibitory function of NKp44 requires further study as it may play a pivotal role in placentation in addition to being exploited by tumors as a mechanism to escape NK cell killing. Finally, we suggest that the NCRs are a class of pattern recognition receptors, which recognize signals of genomic instability and cellular stress via interaction with the c-terminus of DAMP molecules localized to the surface of target cells by various co-ligands.

Keywords: NK cells, natural cytotoxicity receptors, NKp44, DAMPs, tumor ligands

\section{INTRODUCTION}

Natural killer (NK) cells are a fundamental component of the innate immune system, capable of recognizing and destroying tumor cells as well as cells infected by viruses or bacteria (1, 2). NK cells also secrete cytokines such as interferon- $\gamma$ (IFN- $\gamma$ ) and thus regulate the function of other immune cells. Furthermore, NK cells play an important role in adaptive immunity by modulating dendritic cell function and recent findings demonstrate that NK cells have memory $(3,4)$. The ability of NK cells to kill target cells and secrete cytokines is regulated by a delicate balance of activating and inhibitory signals received through distinct classes of receptors found on their cell surface. The balance of signals delivered by those receptors governs NK cell activation, proliferation, and effector functions (5-8). Traditionally, inhibitory killer cell immunoglobulin like receptors (KIRs) and killer cell lectin-like receptors (KLRs) bind cell surface human leukocyte antigen class I (HLA I) molecules expressed by healthy human cells and signal through immunoreceptor tyrosine-based inhibitory motifs (ITIMS) (9-11). When HLA I interacts with inhibitory receptors, dominant inhibitory signaling transmitted by ITIMS prevents activation and cytotoxic action by the NK cell against normal, healthy cells of the body. NK cells may also be inhibited by cytokines released by regulatory cells of the immune system, such as regulatory T cells and myeloid suppressor cells (12).
Activating receptors, including the natural cytotoxicity receptors (NCRs), NKG2D, and 2B4, bind ligands induced by cellular stress, infection, or tumor transformation (13-16). Activating signals are transmitted through immunoreceptor tyrosine-based activating motifs (ITAMs) located in the cytoplasmic tail of the receptor or through ITAMs in adaptor molecules, which associate with activating receptors at the cell surface $(8,17)$. Therefore, when a target cell lacks or under expresses HLA I and/or over expresses activating ligands, NK cells eliminate that target by releasing preformed cytotoxic granzymes and perforin stored as granules or activate apoptosis pathways in the target cell $(8,18)$.

\section{NATURAL CYTOTOXICITY RECEPTORS}

Among the activating receptors is a specialized group of receptors called the NCRs, which play a key role in recognition and killing of tumor and virally infected cells. Comprising the NCRs are the NKp44, NKp30, and NKp46 receptors. Binding of one or more of these receptors with a specific ligand induces strong NK cell activation and cytotoxicity (19). For optimal recognition and elimination of target cells, the NCRs work best as a team when identifying potential targets (20). This is evident through increased cytotoxicity when multiple NCRs are triggered versus an individual receptor, suggesting simultaneous NCR ligand expression on target cells $(20,21)$. Several studies have identified and 
characterized NCR ligands. NKp46 recognition of a ligand on tumor cells has been shown to play a role in prevention of tumor metastasis $(22,23)$. NKp30 is known to bind B7-H6, a member of the B7 family expressed exclusively on tumor cells (24). While many NCR ligands remain unidentified, they are believed not to be expressed by normal cells but induced by cellular stress or pathological conditions (14).

\section{NKp44}

NKp44 is unique and significant for several reasons. First, expression of the receptor is restricted to activated NK cells capable of initiating an immediate cytotoxic response (25). Second, NKp44 activating function is implicated in HIV-related $\mathrm{T}$ cell decline as expression of an activating ligand for NKp44 is induced in uninfected CD4 T cells by the gp41 envelope protein of HIV (26). Earlier studies have shown recognition of viral hemagglutinins of influenza virus by NKp44 enhanced killing of infected cells (27). Finally, NKp44 expression is responsible for a dramatic increase in killing of many tumor cell lines and cross linking the receptor results in the release of cytotoxic granules, IFN- $\gamma$, and TNF- $\alpha$ $(25,28-30)$. While only found on activated NK cells in circulation, NKp44 is constitutively expressed by a specialized subset of NK cells in the decidua, implicating a role for NKp44 during placentation $(25,31,32)$. NKp44 is also expressed on a subset of interferon-producing cells located in human tonsils and ILC3 cells in mucosal-associated lymphoid tissues and human decidua (33-37). Recently, it has been shown that NKp44 is indeed functional in ILC3 and its engagement results in TNF but not in IL-22 production (38).

Crystallographic structure of NKp44 demonstrates a surface groove made by two facing $\beta$ hairpin loops extending from the Ig fold core stabilized by a disulfide bridge between Cystine 37 and Cystine 45 (39). The Ig domain contains an arrangement of positively charged residues at the groove surface, suggesting that NKp44 ligands are anionic (39). Also, the groove appears wide enough to host a sialic acid or an elongated branched ligand. Interestingly, the cytoplasmic tail of NKp44 contains a tyrosine sequence resembling an ITIM $(25,40)$. Contrary to initial reports, this motif is functional and inhibits the release of cytotoxic agents and IFN- $\gamma(25,30,40)$. NKp44 surface expression is dependent on its association with the ITAM containing DAP 12 accessory protein linked to NKp44 through Lysine 183 in the transmembrane domain (25). Upon recognition of activating ligands, signaling transduced through the ITAMs in Dap 12 result in release of cytotoxic agents, tumor necrosis factor- $\alpha$, and IFN- $\gamma(29,40)$.

While NK cells utilize NKp44 to recognize and kill targets, tumors may also exploit NKp44 to escape NK cell recognition. By engaging NKp44, as well as the other NCRs, tumors can induce NK cell death via up regulation of Fas Ligand in the NK cell, inducing Fas-mediated apoptosis (41). Tumors may also downregulate NKp44 surface expression by shedding soluble MHC Class I chainrelated molecules or by releasing indoleamine 2,3-dioxygenase and prostaglandin E2 $(42,43)$. The latter two molecules are released by mesenchymal stem cells as well, inhibiting NKp44 expression in the tumor microenvironment (44). Additionally, tumors can regulate NKp44 ligand expression to escape NK cell killing, as is the case with acute myeloid leukemia (45). Finally, tumor cells may induce expression of exosomal proliferating cell nuclear antigen (PCNA) when physically contacted by NKp44 expressing NK cells to inhibit NK cell effector function (30).

In addition to its role in immunity, $\mathrm{NKp} 44$ also has roles during pregnancy. Decidual NK cells (dNK) make up 50-90\% of lymphocytes in the uterine mucosa during pregnancy and constitutively express NKp44 $(36,46,47)$. Trophoblast cells and maternal stromal cells of the decidua both express unidentified NKp44 ligands (46). This ligand may be PCNA as the protein is over expressed in trophoblast cells during the first trimester (48). As an inhibitory ligand for NKp44, extracellular PCNA expression on trophoblast cells would help explain the diminished ability of dNK cells to lyse trophoblasts despite low levels of classical HLA I expression (47).

\section{NKp44 TUMOR LIGANDS}

NKp44 is implicated in recognition and killing of numerous types of cancer: neuralblastoma, choriocarcinoma, pancreatic, breast, lung adenocarcionma, colon, cervix, hepatocellular carcinoma, Burkitt lymphoma, diffuse B cell lymphoma, prostate $(15,21,28)$. While most of these ligands have not been identified, they appear to be cell cycle regulated, with down regulation of expression during mitosis (28). Recognition of tumor cells is partially mediated through charged-based binding of NKp44 with heparan sulfate proteoglycans (HSPGs) on the surface of tumor cells (49-51). Of note, recognition of HSPG only evokes IFN- $\gamma$ release by NK cells, not cellular cytotoxicity (49). Thus, HSPGs are believed to only be a co-ligand for NKp44 as well as the other NCRs, potentially facilitating binding with other cellular ligands.

Proliferating cell nuclear antigen is the inhibitory tumor ligand for NKp44 $(15,30)$. PCNA is a nuclear protein found in all replicating cells, which encircles DNA and increases processivity of DNA replication, but is also involved in DNA repair and cell cycle control (52). NKp44 recognizes PCNA expressed on exosomes shuttled to the surface of tumors cells when in contact with NK cells $(15,30)$. Recognition of cell surface PCNA colocalizing with HLA I on the cell surface inhibits NK cell cytotoxicity and IFN- $\gamma$ release (15).

A truncated isoform of mixed-lineage leukemia-5 (MLL5) is an activating cellular ligand for NKp44 (53). This MLL5 isoform contains a specific exon encoding a C-terminus, which interacts with NKp44 (53). Typically located only in the nucleus, MLL5 is a lysine methyltransferase implicated in hematopoietic differentiation and control of the cell cycle (53). Contrary to normal MLL5, the isoform recognized by NKp44 is not found in the nucleus but in the cytoplasm and endoplasmic reticulum, destined to be expressed at the cell surface (53). While MLL5 is expressed in normal tissue, the isoform recognized by NKp44 is only present on tumor and transformed cells (53).

\section{NCR CO-LIGANDS}

Heparan sulfate proteoglycans have been identified as co-ligands involved in the recognition of tumor cells by the NCRs $(49,50$, 54). HSPGs are complex glycoproteins found at the cell surface of mammalian cells or in the extracellular matrix $(55,56)$. Heparan sulfate is characterized by chains of disaccharide units of $\mathrm{N}$ acetyl-D-glucosamine linked to D-glucuronic acid $(55,57)$. Interestingly, each NCR recognizes distinct forms of heparan sulfate 
epitopes on HSPGs, specifically highly sulfated microdomains on disaccharide units (58). 2-O-sulfation of iduronic acid and $\mathrm{N}$-acetylation of glucosamine on HSPGs are important for interaction with NKp44 (50). NKp30 and NKp46 recognize HSPGs with 2-O-sulfation of iduronic acid and either 6-O-sulfation or 6$\mathrm{N}$-sulfation of glucosamine (50). Interactions between the NCRs and HSPGs are charge based as each NCR contains basic amino acid residues in their binding cleft and HSPGs are heavily charged molecules.

In addition to HSPGs, HLA I may also serve as a co-ligand for the NCRs. We have shown that HLA I and the NKp44 inhibitory ligand, PCNA, associate on the cell surface (15). In our search to identify a ligand for NKp44, several key pieces of evidence suggested that HLA I plays a role in ligand formation. First, HLA I has been demonstrated to coimmunoprecipitate with anti-NKp44 antibodies; reciprocally, NKp44 coimmunoprecipitates with anti$\beta$-2-microglobulin antibodies (59). Additionally, the Nef protein of HIV prevents surface expression of NKp44 ligand isoform of MLL5 on CD4-infected T cells, which is also consistent with the ability of Nef to retain HLA I intracellularly $(60,61)$. Finally, the NKp30 ligand, Bat3, colocalizes with HLA I on the extracellular membrane of tumor cells, activating NK cell effector functions $(62,63)$. Interestingly, all 50 alleles of HLA Class A, B, and C molecules harbor an Asparagine at position 86, close to the residues on the $\alpha 1$ helix, which determine interactions with human NK receptors (64). This site allows for attachment of N-linked glycan structures, which could enable binding of other proteins (64). Electron density mapping of the HLA I glycan structure suggests that it is flexible and could serve as a ligand for other receptors or block access to HLA I molecules. Additionally, HLA-A and $\mathrm{B}$ are almost exclusively disialylated, resulting in these molecules having a negative charge, a characteristic of NKp44 ligands (64). This negative charge combined with a protruding oligosaccharide could potentially facilitate interactions with NKp44.

As co-ligands, HSPGs and HLA I most likely facilitate binding of other proteins, which together form a complex ligand recognized by the NCRs. The most prevalent interaction between HSPGs and other proteins is charged based via clusters of positively charged amino acids on proteins forming ionic bonds with negatively charged sulfate and carboxyl groups on HSPGs (57). HSPGs may offer two mechanisms facilitating NCR ligand recognition. First, HSPGs may bind soluble proteins, which as a whole serve as ligands for the NCRs. Second, HSPGs could bind a soluble protein and an NCR separately, and then act as scaffolding to bring the NCRs into contact with a soluble protein. In the same manner, the protruding oligosaccharide of HLA I, or other regions, may enable assimilation of small proteins or DAMPs.

\section{DAMPS AND THE C TERMINUS}

Immune responses are initiated by pattern recognition receptors, which recognize microbial-derived products called pathogenassociated molecular pattern molecules (65). In a similar manner, pattern recognition receptors also recognize molecules released by dying or damaged cells, termed damage-associated molecular pattern (DAMP) molecules or alarmins $(66,67)$. Recognition of DAMPs contributes to the induction of inflammation, even in the absence of pathogens (68). Normally residing in the nucleus, cytoplasm, or exosomes, DAMPs lack secretion signals but can be actively secreted by non-classical pathways or passively released by necrotic cells (67). DAMPs thus serve as endogenous danger signals when improperly released from damaged cells as well as tumors and activate innate immune cells (67). DAMPs are most often released after trauma, ischemia, or other tissue damage and initiate early inflammatory responses (67). By recruiting immune cells and promoting the release of proinflammatory mediators, DAMPs activate immune responses and initiate pathways leading to tissue repair and regeneration $(67,69)$. Heat shock proteins, high-mobility group box 1 (HMGB1), S100 proteins, hyaluronan, and heparan sulfate represent a few DAMPs known to date (68).

Like PAMPs, DAMPs are also recognized by pattern recognition receptors. In addition to binding PAMPs, the Toll-like receptors (TLRs) also recognize HMGB1 and a member of the S100 family (67). These two DAMPs are also recognized by the receptor for advanced glycation end products (RAGE) (67). Like the TLRs, RAGE is expressed on numerous immune cells and induces NF$\kappa \mathrm{B}$-mediated production of cytokines (67). Interestingly, DAMP molecules such as high-mobility group protein B1 and S100A8/9 have the ability to bind heparin sulfate and HSPGs, which are known to be co-ligands involved in NCR-dependent recognition of tumor cells resulting in secretion of IFN- $\gamma$ but not cytotoxicity $(49,50,67)$.

We postulate that DAMPs may serve as the missing link in NCRmediated recognition of tumor cells. The association of DAMPs with HSPGs, HLA I, or other potential co-ligands may form larger complex ligands for the members of the NCR family (Figure 1). Human leukocyte antigen-B-associated transcript 3 (Bat3), also known as BAG-6, could be considered a DAMP due to its release from tumor cells (62). Bat3 is typically located in the nucleus where it plays an essential role in controlling the acetylation of p53 in response to cellular DNA damage (62). However, upon non-lethal heat shock, nuclear Bat3 relocates to the cell membrane of tumors where it serves as a ligand for NKp30 $(62,63)$. Interestingly, this study found Bat 3 colocalizes with HLA I, suggesting opposition to previous reports that NCRs do not associate with HLA I molecules $(29,63,70)$.

Like Bat3, PCNA and MLL5 are located in the nucleus and cytoplasm. Additionally, all three molecules are intricately involved in processes regulating the cell cycle and/or DNA repair mechanisms. Thus, their presence on the cell surface may indicate intracellular stress related to DNA damage or improper cell cycle control, qualifying these molecules as DAMPs. This suggests that the NCRs may be pattern recognition receptors, which recognize DAMPs sequestered to the cell surface. Taken into context with other studies, the NCRs potentially have the ability to interact with HLA I, HSPGs, or other cell surface molecules as co-ligands in conjunction with soluble proteins, such as Bat3, PCNA, MLL5, or other DAMPs on the cell surface of tumors (62). Therefore, the NCRs may recognize DAMPs on the cell surface in association with a docking protein. Furthermore, the NCRs may be directly recognizing the C-terminal ends of DAMPs. NKp30 was recently shown to recognize the $\mathrm{C}$ terminus of Bat3 (71). In a similar manner, NKp44 also recognizes the C terminus of the MLL5 ligand (53). The molecular details of interaction between NKp44 and PCNA 

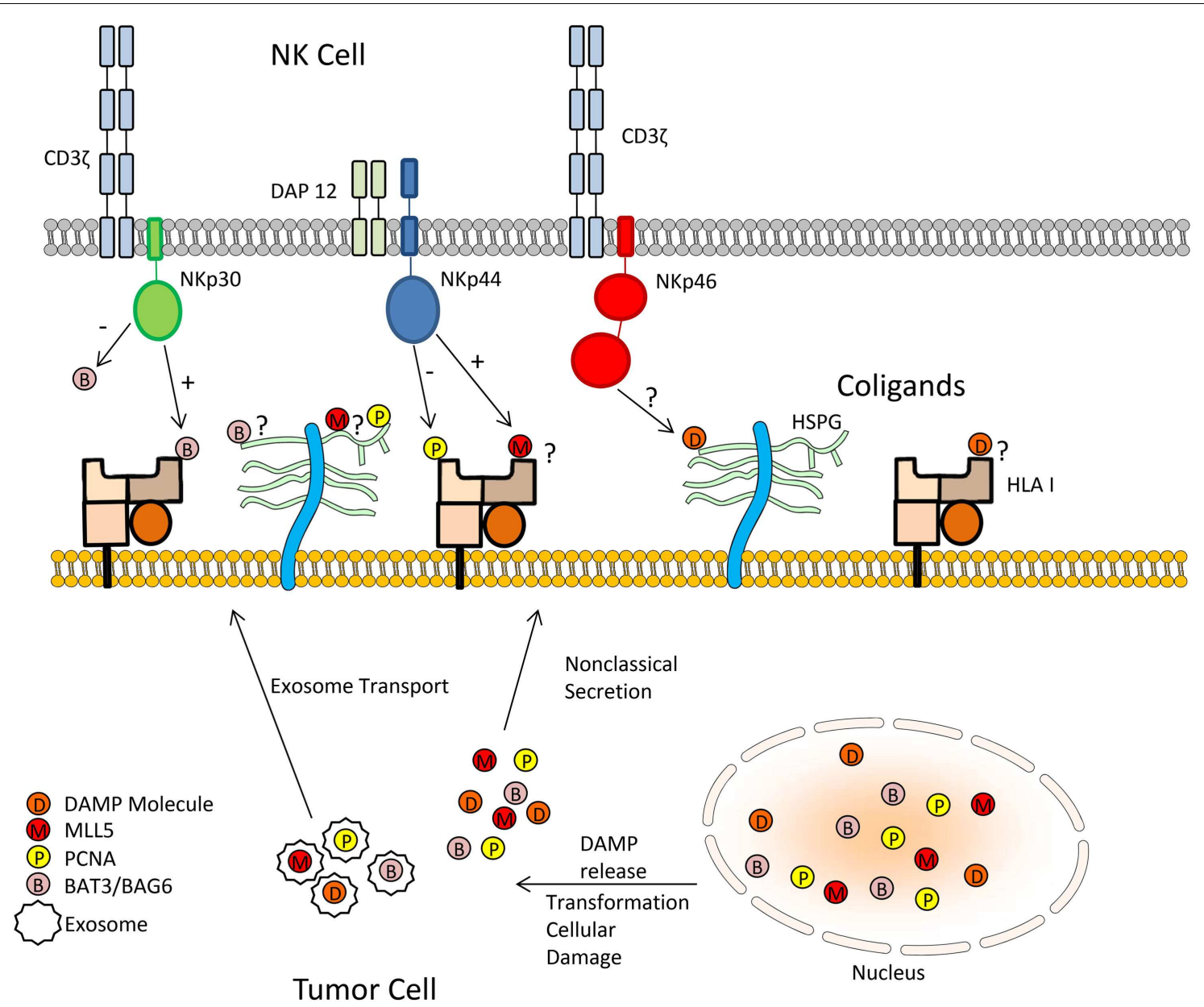

FIGURE 1 | Mechanism by which tumor cells may inhibit natural cytotoxicity through NCR recognition by DAMPs. The association of DAMPs with HSPGs, HLA I, or other potential co-ligands may form larger complex ligands for the members of the NCR family.

have yet to be determined, but the above precedents suggest that interaction may occur at the $\mathrm{C}$ terminus of PCNA.

Natural cytotoxicity receptors ligands consisting of a DAMP and a co-ligand may add complexity in understanding how the NCRs regulate NK cell effector function. The NCRs were originally believed to be strictly activating NK cell receptors. However, NKp44 and NKp30 have recently been shown to exhibit both inhibitory and activating functions. NKp44 recognizes cell surface PCNA in an inhibitory manner while a soluble C-terminal fragment of Bat3 inhibits NK cell function via $\operatorname{NKp30}(15,71)$. However, recognition of MLL5 by NKp44- and NKp30-mediated recognition of Bat3 sequestered to the cell surface activates NK cell effector functions $(53,62,63)$. Thus, modulation of NK cell activity via the NCRs could depend on the DAMP molecule, the co-ligand sequestering the DAMP, or the lack of a coligand and the soluble nature of the DAMP. NKp44 presents a more special case since it contains a functional ITIM-like sequence in its cytoplasmic tail. Due to the dual nature of NKp44 signaling, it will be of interest to determine if recognition of the DAMP, either PCNA or MLL5, the coligand, potentially an HSPG or HLA I, or the motif as a whole is responsible for inhibition or activation of NK cytotoxicity. Neither NKp30 nor NKp46 has been reported to contain an ITIM sequence. However, an immunosuppressive isoform of NKp30 resulting from a single-nucleotide polymorphism in the $3^{\prime}$-untranslatable region has been reported (72). Whether the divergence of NCR function depends on the individual DAMP molecule recognized or the binding of DAMP molecules to a specific coligand remains to be elucidated.

\section{CONCLUDING REMARIKS}

Recent studies reveal a novel function for DAMP molecules, or proteins, which are located and function intracellular, but somehow localize to the extracellular membrane despite lacking a traditional secretory leader sequence. These proteins are released by cells, which have become injured in the absence of infection due to ischemia, hypoxia, transformation, chemotherapy, DNA damage, or other trauma. Analogous to TLRs recognizing pathogenassociated molecular patterns, the NCRs may represent a class of NK cell receptors that participate in pattern recognition of DAMP molecules, whose identities may reflect the intracellular health of a 
cell, particularly in regards to DNA damage or instability, in addition to the traditional method of HLA I presenting self peptide. In this manner, HLA I, HSPGs, or other co-ligands may present DAMP molecules for identification by the NCRs, which would then regulate NK cell function. Like other NK cell receptors, the NCRs undoubtedly recognize multiple ligands, which may be cell surface transmembrane proteins, like the recognition of B7-H6 molecule by NKp30 (73). Knowledge of the identities of NCR ligands and nature of DAMP molecules that bind to HLA I, HSPGs, or other cell surface molecules to form complex ligands for the NCRs will shed light on NK cell recognition of target cells under healthy and disease conditions and offer novel therapeutic targets.

\section{ACKNOWLEDGMENTS}

This work was supported by UNTHSC Seed Grant (Mathew).

\section{REFERENCES}

1. Lanier LL. Up on the tightrope: natural killer cell activation and inhibition. Nat Immunol (2008) 9:495-502. doi:10.1038/ni1581

2. Biron CA, Nguyen KB, Pien GC, Cousens LP, Salazar-Mather TP. Natural killer cells in antiviral defense: function and regulation by innate cytokines. Annu Rev Immunol (1999) 17:189-220. doi:10.1146/annurev.immunol.17.1.189

3. Ferlazzo G, Moretta L. Dendritic cell editing by natural killer cells. Crit Rev Oncog (2014) 19:67-75. doi:10.1615/CritRevOncog.2014010827

4. Min-Oo G, Kamimura Y, Hendricks DW, Nabekura T, Lanier LL. Natural killer cells: walking three paths down memory lane. Trends Immunol (2013) 34:251-8. doi:10.1016/j.it.2013.02.005

5. Lanier LL. NK cell recognition. Annu Rev Immunol (2005) 23:225-74. doi:10. 1146/annurev.immunol.23.021704.115526

6. Colonna M, Jonjic S, Watzl C. Natural killer cells: fighting viruses and much more. Nat Immunol (2011) 12:107-10. doi:10.1038/ni0211-107

7. Yokoyama WM, Kim S, French AR. The dynamic life of natural killer cells. Annu Rev Immunol (2004) 22:405-29. doi:10.1146/annurev.immunol.22. 012703.104711

8. Lanier LL, Phillips JH. NK cell recognition of major histocompatibility complex class I molecules. Semin Immunol (1995) 7:75-82. doi:10.1006/smim.1995.0011

9. Long EO. Negative signaling by inhibitory receptors: the NK cell paradigm. Immunol Rev (2008) 224:70-84. doi:10.1111/j.1600-065X.2008.00660.x

10. Lanier LL. Missing self, NK cells, and the white album. J Immunol (2005) 174:6565. doi:10.4049/jimmunol.174.11.6565

11. Moretta L, Bottino C, Pende D, Castriconi R, Mingari MC, Moretta A. Surface NK receptors and their ligands on tumor cells. Semin Immunol (2006) 18:151-8. doi:10.1016/j.smim.2006.03.002

12. Barao I, Hanash AM, Hallett W, Welniak LA, Sun K, Redelman D, et al. Suppression of natural killer cell-mediated bone marrow cell rejection by CD4+CD25+ regulatory T cells. Proc Natl Acad Sci U S A (2006) 103:5460-5. doi:10.1073/pnas.0509249103

13. Cerwenka A, Lanier LL. Ligands for natural killer cell receptors: redundancy or specificity. Immunol Rev (2001) 181:158-69. doi:10.1034/j.1600-065X.2001. 1810113.x

14. Bottino C, Castriconi R, Moretta L, Moretta A. Cellular ligands of activating NK receptors. Trends Immunol (2005) 26:221-6. doi:10.1016/j.it.2005.02.007

15. Horton NC, Mathew SO, Mathew PA. Novel interaction between proliferating cell nuclear antigen and HLA I on the surface of tumor cells inhibits NK cell function through NKp44. PLoS One (2013) 8:e59552. doi:10.1371/journal.pone. 0059552

16. Boles KS, Stepp SE, Bennett M, Kumar V, Mathew PA. 2B4 (CD244) and CS1: novel members of the CD2 subset of the immunoglobulin superfamily molecules expressed on natural killer cells and other leukocytes. Immunol Rev (2001) 181:234-49. doi:10.1034/j.1600-065X.2001.1810120.x

17. Cerwenka A, Lanier LL. Natural killer cells, viruses and cancer. Nat Rev Immunol (2001) 1:41-9. doi:10.1038/35095564

18. Moretta L, Moretta A. Unravelling natural killer cell function: triggering and inhibitory human NK receptors. EMBO J (2004) 23:255-9. doi:10.1038/sj. emboj.7600019
19. Kruse PH, Matta J, Ugolini S, Vivier E. Natural cytotoxicity receptors and their ligands. Immunol Cell Biol (2014) 92:221-9. doi:10.1038/icb.2013.98

20. Augugliaro R, Parolini S, Castriconi R, Marcenaro E, Cantoni C, Nanni M, et al. Selective cross-talk among natural cytotoxicity receptors in human natural killer cells. Eur J Immunol (2003) 33:1235-41. doi:10.1002/eji.200323896

21. Sivori S, Parolini S, Marcenaro E, Castriconi R, Pende D, Millo R, et al. Involvement of natural cytotoxicity receptors in human natural killer cell-mediated lysis of neuroblastoma and glioblastoma cell lines. J Neuroimmunol (2000) 107:220-5. doi:10.1016/S0165-5728(00)00221-6

22. Glasner A, Ghadially H, Gur C, Stanietsky N, Tsukerman P, Enk J, et al. Recognition and prevention of tumor metastasis by the NK receptor NKp46/NCR1. J Immunol (2012) 188:2509-15. doi:10.4049/jimmunol.1102461

23. Cagnano E, Hershkovitz O, Zilka A, Bar-Ilan A, Golder A, Sion-Vardy N, et al. Expression of ligands to NKp46 in benign and malignant melanocytes. J Invest Dermatol (2008) 128:972-9. doi:10.1038/sj.jid.5701111

24. Koch J, Steinle A, Watzl C, Mandelboim O. Activating natural cytotoxicity receptors of natural killer cells in cancer and infection. Trends Immunol (2013) 34:182-91. doi:10.1016/j.it.2013.01.003

25. Cantoni C, Bottino C, Vitale M, Pessino A, Augugliaro R, Malaspina A, et al. $\mathrm{NKp44}$, a triggering receptor involved in tumor cell lysis by activated human natural killer cells, is a novel member of the immunoglobulin superfamily. J Exp Med (1999) 189:787-96. doi:10.1084/jem.189.5.787

26. Vieillard V, Strominger JL, Debre P. NK cytotoxicity against CD4+ T cells during HIV-1 infection: a gp41 peptide induces the expression of an NKp44 ligand. Proc Natl Acad Sci U S A (2005) 102:10981-6. doi:10.1073/pnas.0504315102

27. Arnon TI, Lev M, Katz G, Chernobrov Y, Porgador A, Mandelboim O. Recognition of viral hemagglutinins by NKp44 but not by NKp30. Eur J Immunol (2001) 31:2680-9. doi:10.1002/1521-4141(200109)31:9<2680::AID-IMMU2680>3.0. CO;2-A

28. Byrd A, Hoffmann SC, Jarahian M, Momburg F, Watzl C. Expression analysis of the ligands for the natural killer cell receptors NKp30 and NKp44. PLoS One (2007) 2:e1339. doi:10.1371/journal.pone.0001339

29. Vitale M, Bottino C, Sivori S, Sanseverino L, Castriconi R, Marcenaro E, et al. NKp44, a novel triggering surface molecule specifically expressed by activated natural killer cells, is involved in non-major histocompatibility complexrestricted tumor cell lysis. J Exp Med (1998) 187:2065-72. doi:10.1084/jem.187. 12.2065

30. Rosental B, Brusilovsky M, Hadad U, Oz D, Appel MY, Afergan F, et al. Proliferating cell nuclear antigen is a novel inhibitory ligand for the natural cytotoxicity receptor NKp44. J Immunol (2011) 187:5693-702. doi:10.4049/jimmunol. 1102267

31. Manaster I, Mandelboim O. The unique properties of uterine NK cells. Am J Reprod Immunol (2010) 63:434-44. doi:10.1111/j.1600-0897.2009.00794.x

32. de Rham C, Ferrari-Lacraz S, Jendly S, Schneiter G, Dayer JM, Villard J. The proinflammatory cytokines IL-2, IL-15 and IL-21 modulate the repertoire of mature human natural killer cell receptors. Arthritis Res Ther (2007) 9:R125. doi:10.1186/ar2336

33. Cella M, Fuchs A, Vermi W, Facchetti F, Otero K, Lennerz JK, et al. A human natural killer cell subset provides an innate source of IL-22 for mucosal immunity. Nature (2009) 457:722-5. doi:10.1038/nature07537

34. Cella M, Otero K, Colonna M. Expansion of human NK-22 cells with IL-7, IL-2, and IL-1beta reveals intrinsic functional plasticity. Proc Natl Acad Sci U S A (2010) 107:10961-6. doi:10.1073/pnas.1005641107

35. Fuchs A, Cella M, Kondo T, Colonna M. Paradoxic inhibition of human natural interferon-producing cells by the activating receptor NKp44. Blood (2005) 106:2076-82. doi:10.1182/blood-2004-12-4802

36. Vacca P, Montaldo E, Croxatto D, Loiacono F, Canegallo F, Venturini PL, et al. Identification of diverse innate lymphoid cells in human decidua. Mucosal Immunol (2014). doi:10.1038/mi.2014.63

37. Spits H, Artis D, Colonna M, Diefenbach A, Di Santo JP, Eberl G, et al. Innate lymphoid cells - a proposal for uniform nomenclature. Nat Rev Immunol (2013) 13:145-9. doi:10.1038/nri3365

38. Glatzer T, Killig M, Meisig J, Ommert I, Luetke-Eversloh M, Babic M, et al. RORgammat $(+)$ innate lymphoid cells acquire a proinflammatory program upon engagement of the activating receptor NKp44. Immunity (2013) 38:1223-35. doi:10.1016/j.immuni.2013.05.013

39. Cantoni C, Ponassi M, Biassoni R, Conte R, Spallarossa A, Moretta A, et al. The three-dimensional structure of the human NK cell receptor NKp44, a 
triggering partner in natural cytotoxicity. Structure (Camb) (2003) 11:725-34. doi:10.1016/S0969-2126(03)00095-9

40. Campbell KS, Yusa S, Kikuchi-Maki A, Catina TL. NKp44 triggers NK cell activation through DAP12 association that is not influenced by a putative cytoplasmic inhibitory sequence. J Immunol (2004) 172:899-906. doi:10.4049/jimmunol. 172.2.899

41. Poggi A, Massaro AM, Negrini S, Contini P, Zocchi MR. Tumor-induced apoptosis of human IL-2-activated NK cells: role of natural cytotoxicity receptors. $J$ Immunol (2005) 174:2653-60. doi:10.4049/jimmunol.174.5.2653

42. Doubrovina ES, Doubrovin MM, Vider E, Sisson RB, O’Reilly RJ, Dupont B, et al. Evasion from NK cell immunity by MHC class I chain-related molecules expressing colon adenocarcinoma. J Immunol (2003) 171:6891-9. doi:10.4049/ jimmunol.171.12.6891

43. Pietra G, Manzini C, Rivara S, Vitale M, Cantoni C, Petretto A, et al. Melanoma cells inhibit natural killer cell function by modulating the expression of activating receptors and cytolytic activity. Cancer Res (2012) 72:1407-15. doi:10.1158/ 0008-5472.CAN-11-2544

44. Spaggiari GM, Capobianco A, Abdelrazik H, Becchetti F, Mingari MC, Moretta L. Mesenchymal stem cells inhibit natural killer cell proliferation, cytotoxicity and cytokine production: role of indoleamine 2,3-dioxygenase and prostaglandin E2. Blood (2007) 111(3):1327-33. doi:10.1182/blood-2007-02-074997

45. Nowbakht P, Ionescu MC, Rohner A, Kalberer CP, Rossy E, Mori L, et al. Ligands for natural killer cell-activating receptors are expressed upon the maturation of normal myelomonocytic cells but at low levels in acute myeloid leukemias. Blood (2005) 105:3615-22. doi:10.1182/blood-2004-07-2585

46. Hanna J, Goldman-Wohl D, Hamani Y, Avraham I, Greenfield C, NatansonYaron S, et al. Decidual NK cells regulate key developmental processes at the human fetal-maternal interface. Nat Med (2006) 12:1065-74. doi:10.1038/ nm1452

47. Vacca P, Cantoni C, Prato C, Fulcheri E, Moretta A, Moretta L, et al. Regulatory role of NKp44, NKp46, DNAM-1 and NKG2D receptors in the interaction between NK cells and trophoblast cells. Evidence for divergent functional profiles of decidual versus peripheral NK cells. Int Immunol (2008) 20:1395-405. doi:10.1093/intimm/dxn105

48. Korgun ET, Celik-Ozenci C, Acar N, Cayli S, Desoye G, Demir R. Location of cell cycle regulators cyclin B1, cyclin A, PCNA, Ki67 and cell cycle inhibitors p21, p27 and p57 in human first trimester placenta and deciduas. Histochem Cell Biol (2006) 125:615-24. doi:10.1007/s00418-006-0160-y

49. Hershkovitz O, Jivov S, Bloushtain N, Zilka A, Landau G, Bar-Ilan A, et al. Characterization of the recognition of tumor cells by the natural cytotoxicity receptor, NKp44. Biochemistry (2007) 46:7426-36. doi:10.1021/bi7000455

50. Hecht ML, Rosental B, Horlacher T, Hershkovitz O, De Paz JL, Noti C, et al. Natural cytotoxicity receptors NKp30, NKp44 and NKp46 bind to different heparan sulfate/heparin sequences. J Proteome Res (2009) 8:712-20. doi: $10.1021 / \mathrm{pr} 800747 \mathrm{c}$

51. Arase H, Mocarski ES, Campbell AE, Hill AB, Lanier LL. Direct recognition of cytomegalovirus by activating and inhibitory NK cell receptors. Science (2002) 296:1323-6. doi:10.1126/science.1070884

52. Maga G, Hubscher U. Proliferating cell nuclear antigen (PCNA): a dancer with many partners. J Cell Sci (2003) 116:3051-60. doi:10.1242/jcs.00653

53. Baychelier F, Sennepin A, Ermonval M, Dorgham K, Debré P, Vieillard V. Identification of a cellular ligand for the natural cytotoxicity receptor NKp44. Blood (2013) 122(17):2935-42. doi:10.1182/blood-2013-03-489054

54. Brusilovsky M, Radinsky O, Yossef R, Campbell KS, Porgador A. Carbohydratemediated modulation of NK cell receptor function: structural and functional influences of heparan sulfate moieties expressed on NK cell surface. Front Oncol (2014) 4:185. doi:10.3389/fonc.2014.00185

55. Sasisekharan R, Venkataraman G. Heparin and heparan sulfate: biosynthesis, structure and function. Curr Opin Chem Biol (2000) 4:626-31. doi:10.1016/ S1367-5931(00)00145-9

56. Knelson EH, Nee JC, Blobe GC. Heparan sulfate signaling in cancer. Trends Biochem Sci (2014) 39:277-88. doi:10.1016/j.tibs.2014.03.001

57. Xu D, Esko JD. Demystifying heparan sulfate-protein interactions. Annu Rev Biochem (2014) 83:129-57. doi:10.1146/annurev-biochem-060713-035314

58. Brusilovsky M, Rosental B, Shemesh A, Appel MY, Porgador A. Human NK cell recognition of target cells in the prism of natural cytotoxicity receptors and their ligands. J Immunotoxicol (2012) 9:267-74. doi:10.3109/1547691X.2012. 675366
59. Betser-Cohen G, Mizrahi S, Elboim M, Alsheich-Bartok O, Mandelboim O. The association of MHC class I proteins with the 2B4 receptor inhibits self-killing of human NK cells. J Immunol (2010) 184:2761-8. doi:10.4049/jimmunol.0901572

60. Fausther-Bovendo H, Sol-Foulon N, Candotti D, Agut H, Schwartz O, Debré $\mathrm{P}$, et al. HIV escape from natural killer cytotoxicity: nef inhibits NKp44L expression on CD4+ T cells. AIDS (2009) 23:1077-87. doi:10.1097/QAD. 0b013e32832cb26b

61. Specht A, DeGottardi MQ, Schindler M, Hahn B, Evans DT, Kirchhoff F. Selective downmodulation of HLA-A and -B by Nef alleles from different groups of primate lentiviruses. Virology (2008) 373:229-37. doi:10.1016/j.virol.2007.11.019

62. Pogge von Strandmann E, Simhadri VR, von Tresckow B, Sasse S, Reiners KS et al. Human leukocyte antigen-B-associated transcript 3 is released from tumor cells and engages the NKp30 receptor on natural killer cells. Immunity (2007) 27:965-74. doi:10.1016/j.immuni.2007.10.010

63. Simhadri VR, Reiners KS, Hansen HP, Topolar D, Simhadri VL, Nohroudi K, et al. Dendritic cells release HLA-B-associated transcript-3 positive exosomes to regulate natural killer function. PLoS One (2008) 3:e3377. doi:10.1371/journal. pone. 0003377

64. Barber LD, Patel TP, Percival L, Gumperz JE, Lanier LL, Phillips JH, et al. Unusual uniformity of the N-linked oligosaccharides of HLA-A, -B, and -C glycoproteins. J Immunol (1996) 156:3275-84.

65. Janeway CA Jr, Medzhitov R. Innate immune recognition. Annu Rev Immuno (2002) 20:197-216. doi:10.1146/annurev.immunol.20.083001.084359

66. Lotze MT, Zeh HJ, Rubartelli A, Sparvero LJ, Amoscato AA, Washburn NR, et al. The grateful dead: damage-associated molecular pattern molecules and reduction/oxidation regulate immunity. Immunol Rev (2007) 220:60-81. doi:10.1111/ j.1600-065X.2007.00579.x

67. Srikrishna G, Freeze HH. Endogenous damage-associated molecular pattern molecules at the crossroads of inflammation and cancer. Neoplasia (2009) 11:615-28. doi:10.1593/neo.09284

68. Rubartelli A, Lotze MT. Inside, outside, upside down: damage-associated molecular-pattern molecules (DAMPs) and redox. Trends Immunol (2007) 28:429-36. doi:10.1016/j.it.2007.08.004

69. Uchida K. Natural antibodies as a sensor of electronegative damage-associated molecular patterns (DAMPs). Free Radic Biol Med (2014) 72:156-61. doi:10. 1016/j.freeradbiomed.2014.03.016

70. Moretta A, Bottino C, Vitale M, Pende D, Cantoni C, Mingari MC, et al. Activating receptors and coreceptors involved in human natural killer cellmediated cytolysis. Annu Rev Immunol (2001) 19:197-223. doi:10.1146/ annurev.immunol.19.1.197

71. Binici J, Hartmann J, Herrmann J, Schreiber C, Beyer S, Güler G, et al. A soluble fragment of the tumor antigen BCL2-associated athanogene 6 (BAG-6) is essential and sufficient for inhibition of NKp30 receptor-dependent cytotoxicity of natural killer cells. J Biol Chem (2013) 288:34295-303. doi:10.1074/jbc.M113. 483602

72. Delahaye NF, Rusakiewicz S, Martins I, Ménard C, Roux S, Lyonnet L, et al. Alternatively spliced $\mathrm{NKp} 30$ isoforms affect the prognosis of gastrointestinal stromal tumors. Nat Med (2011) 17:700-7. doi:10.1038/nm.2366

73. Brandt CS, Baratin M, Yi EC, Kennedy J, Gao Z, Fox B, et al. The B7 family member B7-H6 is a tumor cell ligand for the activating natural killer cell receptor NKp30 in humans. J Exp Med (2009) 206:1495-503. doi:10.1084/jem.20090681

Conflict of Interest Statement: The authors declare that the research was conducted in the absence of any commercial or financial relationships that could be construed as a potential conflict of interest.

Received: 10 November 2014; accepted: 15 January 2015; published online: 02 February 2015 .

Citation: Horton NC and Mathew PA (2015) NKp44 and natural cytotoxicity receptors as damage-associated molecular pattern recognition receptors. Front. Immunol. 6:31. doi: 10.3389/fimmu.2015.00031

This article was submitted to NK Cell Biology, a section of the journal Frontiers in Immunology.

Copyright (c) 2015 Horton and Mathew. This is an open-access article distributed under the terms of the Creative Commons Attribution License (CC BY). The use, distribution or reproduction in other forums is permitted, provided the original author (s) or licensor are credited and that the original publication in this journal is cited, in accordance with accepted academic practice. No use, distribution or reproduction is permitted which does not comply with these terms. 